\title{
Transport in Dense Nuclear Matter
}

\section{Stephan Stetina*}

Institute for Theoretical Physics, Vienna University of Technology

E-mail: stetina@hep.itp.tuwien.ac.at

Dense nuclear matter in the core of neutron stars consists, in its simplest manifestation, of a plasma of degenerate neutrons, protons, electrons, and muons in $\beta$ equilibrium. I present a calculation of the scattering rates of electrons and muons, taking into account correlations of strong and electromagnetic interactions. These correlations allow for medium-induced lepton-neutron scattering, which in turn leads to a sizeable modification of the total scattering rate. The impact of induced interactions is particularly pronounced close to the crust-core boundary of neutron stars, which makes the results potentially relevant for the damping of hydrodynamic modes and r-modes.

XIII Quark Confinement and the Hadron Spectrum - Confinement2018

31 July - 6 August 2018

Maynooth University, Ireland

${ }^{*}$ Speaker. 


\section{Introduction}

The properties of cold and dense matter in the core of neutron stars are determined by an interplay of strong, electromagnetic, and weak interactions. A rich phase structure, shaped by the density dependence of strong interactions is projected to emerge, including the possible occurrence of superconductivity and superfluidity, hyperons, meson condensates, and deconfined quark matter. Depending on the microscopic properties of each of these phases, transport can be greatly enhanced or suppressed, see Ref. [1] for a recent review.

In this article, I focus on the outer core of neutron stars, i.e., on a region where densities are just high enough to support stable homogeneous nuclear matter, leaving aside more exotic phases that may reside deeper in the core. Electrons, and, depending on the density also muons, can be regarded as quasiparticles immersed in a weekly interacting degenerate plasma. Nucleons can be described quasiparticles in a strongly interacting Fermi liquid. To obtain the relative abundance of each fermion species, the conditions imposed by charge neutrality and $\beta$ equilibrium are employed, typically resulting in highly asymmetric nuclear matter, with proton fractions below $10 \%$. Transport in degenerate matter is computed from the scattering rates of particles in close proximity to the Fermi surface. The dispersion relations of weakly interacting particles are modified by collective effects if their energies are small compared to the Fermi energy. For all practical purposes, one may thus use free, on-shell dispersion relations for electrons and muons. In the case of nucleons, however, the knowledge of effective masses $m_{p, n}^{*}$ and residual quasiparticle interactions in close proximity to the Fermi surface is critical. Both quantities can be related to Fermi-liquid parameters in the low energy limit, which in the present case are in turn related to Skyrme type nuclear interactions. While the model dependence of Skyrme forces increases with density, they are fairly well constrained at densities of interest to this article. It should be noted, that attractive s-wave interactions are expected to result in proton superconductivity in the outer core, although recent calculations taking into account three-nucleon interactions seem to expose it as a much more fragile phenomenon than previously assumed [2]. In the present analysis I neglect effects due to Cooper pairing of nucleons, which implies that the results obtained are not valid at very low temperatures $T<T_{c}$, where $T_{c}$ is the critical temperature of superconductivity. However, the remaining range of temperatures available in neutron stars, $T_{c}<T \lesssim 1 \mathrm{MeV}$, is still tiny compared to the chemical potentials of leptons and nucleons, such that a fully degenerate calculation is well justified.

In the environment described above, electrons are near-ultrarelativistic, which makes them a dominant contributor to transport phenomena, and the focal point of this article. The lepton contribution to transport has been calculated in Refs. [3] and [4], and in Ref. [5] taking into account the effects of proton superconductivity. An outstanding question is the role of neutrons in lepton scattering. In earlier work electron-neutron scattering due to the weak magnetic moment of the neutron was considered, and deemed unimportant [6]. Recently, a new scattering mechanism of electrons and neutrons, induced by the polarizability of electromagnetically and strongly charged protons has been pointed out in Ref. [7]. A consistent resummation of these induced interactions to obtain the dressed photon propagator has been carried out [8], and the results indicate that neutrons may indeed strongly modify electromagnetic scattering at lower densities. The total scattering rates of particles in close proximity to the crust-core boundary of neutron stars is particularly relevant for the damping of hydrodynamic modes and r-modes. The excitation of r-modes in fast spinning stars 

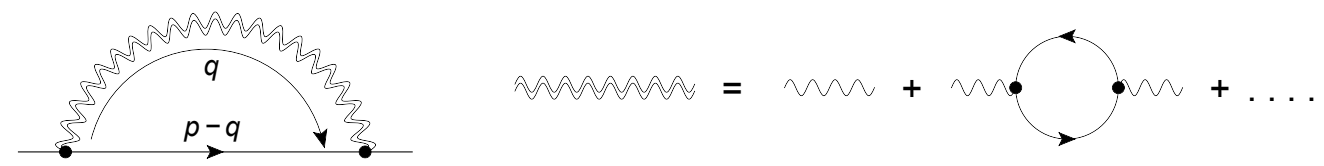

Figure 1: Leading contribution to the self energy $\Sigma$ of a fermion thermalized in a degenerate QED plasma. The photon propagator needs to be dressed, in the simplest case by single loop insertions. This is commonly referred to as Random Phase Approximation (RPA).

seems unavoidable, yet they are known to become unstable with respect to the emission of gravitational waves [9]. The fact that fast spinning stars are observed in nature points towards an efficient damping mechanism. If viscous damping is responsible for the stabilization of fast spinning stars, it would have to be several times larger than previously calculated.

In the following I briefly outline the calculation of scattering rates of electrons and muons based on the Random Phase Approximation (RPA), taking into account correlations of electromagnetic and strong interactions. To extract the properties of the nucleon quasiparticles, I use the same set of modern Skyrme forces as in Ref. [8], including NRAPR, SKRA, SQMC700, LNS, and KDE0v1. These models predict the stability of homogeneous nuclear matter at densities above $n_{c} \sim(0.54-0.59) n_{0}$. The results presented cover densities from $n_{c}$ till $2 n_{0}$, where $n_{0}$ is the nuclear saturation density.

\section{Calculation of the scattering rate}

By virtue of the optical theorem, the total rate of Moeller scattering can be obtained from the imaginary part of the fermion self-energy. The generic relationship reads

$$
\Gamma(\mathbf{p})=-\frac{1}{2 \varepsilon_{\mathbf{p}}}\left[1-n_{f}\left(\boldsymbol{\varepsilon}_{\mathbf{p}}\right)\right] \operatorname{Tr}\left[(p+m) \operatorname{Im} \Sigma_{R}\left(p_{0}=\varepsilon_{\mathbf{p}}, \mathbf{p}\right)\right],
$$

where $\varepsilon_{p}=\sqrt{\mathbf{p}^{2}+m^{2}}$, and $\Sigma_{R}$ is the retarded self energy. The scattering rate of holes can be recovered upon replacing the factor $1-n_{f}$ by $n_{f}$. Note, that, as mentioned before, we have assumed that electrons and muons carry momenta of order $k_{f}$, and have consequently put them on-shell in equation 2.1. The energy $q_{0}$ of the exchanged photon may well be small compared to $k_{f}$, and medium corrections to the photon propagator are essential in the calculation of $\Sigma_{R}$. A diagramatic representation of the relevant contribution is depicted in Fig. 1. It should be remarked, that the interference contributions to $t$ and $u$ channel scattering of identical fermions cannot be extracted from the self-energy diagram shown in Fig. 1. In any case interference terms have been shown to be small for leptons under neutron star conditions [4]. Evaluating Eq. 2.1 using a spectral representation of the dressed photon propagator one finds (in Coulomb gauge)

$$
\Gamma_{L, \perp}\left(\varepsilon_{\mathbf{p}}\right)=\frac{e^{2}}{2}\left[1-n_{f}\left(\varepsilon_{\mathbf{p}}\right)\right] \int \frac{d^{3} \mathbf{q}}{(2 \pi)^{2}} \rho_{L, \perp}\left(\varepsilon_{\mathbf{p}}-\varepsilon_{\mathbf{p}^{\prime}}, \mathbf{q}\right)\left[1+n_{b}\left(\varepsilon_{\mathbf{p}}-\varepsilon_{\mathbf{p}^{\prime}}\right)-n_{f}^{-}\left(\varepsilon_{\mathbf{p}^{\prime}}\right)\right] K_{L, \perp},
$$

with the coefficients

$$
K_{L}=1+\frac{\varepsilon_{\mathbf{p}}^{2}-\mathbf{p} \cdot \mathbf{q}}{\varepsilon_{\mathbf{p}} \varepsilon_{\mathbf{p}^{\prime}}}, \quad K_{\perp}=2\left[1+\frac{\mathbf{p}_{\perp}^{2}-\varepsilon_{\mathbf{p}}^{2}+\mathbf{p} \cdot \mathbf{q}}{\varepsilon_{\mathbf{p}} \varepsilon_{\mathbf{p}^{\prime}}}\right] .
$$




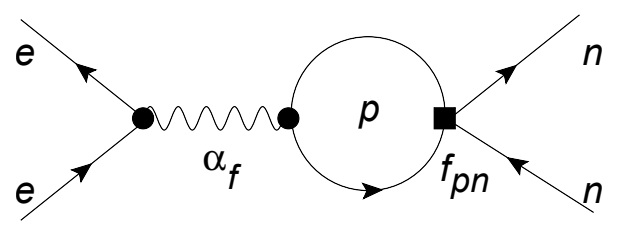

Figure 2: Lepton-neutron scattering, mediated by the polarizability of strongly and electromagnetically charged protons. The squared vertex denotes the residual interaction of the nucleon quasiparticles.

There are now two scattering channels, governed by the exchange transverse photons and longitudinal plasmons. The latter is a pure collective excitation and disappears from the spectrum upon increasing the photon momentum $q$. The respective spectral functions can be obtained from the resummed photon propagator

$$
\rho_{L}(q)=-\frac{1}{\pi} \frac{\operatorname{Im} \Pi_{L}}{\left(\operatorname{Re} \Pi_{L}-\mathbf{q}^{2}\right)^{2}+\left(\operatorname{Im} \Pi_{L}\right)^{2}}, \quad \rho_{\perp}(q)=-\frac{1}{\pi} \frac{\operatorname{Im} \Pi_{\perp}}{\left(\operatorname{Re} \Pi_{\perp}-q^{2}\right)^{2}+\left(\operatorname{Im} \Pi_{\perp}\right)^{2}},
$$

where $\Pi=\Pi_{R}\left(q_{0}<|\mathbf{q}|\right)$ is the retarded one-loop photon polarization tensor, evaluated in the spacelike domain. In the time-like region the spectral function does not contribute to the scattering process. In each channel interactions are screened by medium effects, albeit in a very distinct manner. Scattering in the longitudinal channel is predominantly modified by Debye screening, stemming from the real part of $\Pi_{L}$. Debye screening persists even in the static limit, where one obtains the screening mass as $\Pi_{L}\left(q_{0},|\mathbf{q}| \rightarrow 0\right)=-m_{D}^{2}$. The transverse polarization tensor vanishes in the static limit. The dominant contribution to (dynamical) screening then originates from Landau damping, embodied in the imaginary part of $\Pi_{\perp}$. Taking into account these characteristics, Heiselberg and Pethick realized [10], that the scattering of ultra relativistic particles is dominated by the exchange of transverse photons, while the scattering of non-relativistic particles is dominated by the exchange of longitudinal plasmons. In the present context, ultra-relativistic particles are represented by electrons, non-relativistic particles are represented by nucleons (at least at lower densities), and muons interpolate between both scenarios. In the degenerate limit the evaluation of Eq. 2.2 is facilitated by the replacements $n_{f}\left(\varepsilon_{\mathbf{p}}\right) \rightarrow \theta\left(\mu-\varepsilon_{\mathbf{p}}\right)$, and $1+n_{b}\left(q_{0}\right) \rightarrow \theta\left(q_{0}\right)$. In case of $\Gamma_{\perp}$, strict Pauli blocking is essential to obtain a finite result. At finite temperature, Landau damping is insufficient to screen the Rutherford singularity in the infrared.

Within the RPA, it is particularly simple to generalize the scattering rate to a multi-component plasma composed of electrons, muons, and protons (EMP plasma). The photon polarization tensor in Eq. 2.4 is replaced by the sum of the individual polarizations, $\Pi=\Pi_{e}+\Pi_{\mu}+\Pi_{p}$, where the chemical potentials of each particle species are fixed by the requirements of $\beta$ equilibrium and charge neutrality. The inclusion of neutrons (EMPN plasma) is a bit more subtle. In free space, lepton-neutron scattering arises only due to the small magnetic moment of the neutron, and has a negligible impact on the total scattering rate. In nuclear matter, electromagnetically and strongly charged protons mediate interactions between leptons and neutrons, see Fig. 2. The consistent resummation of induced interactions to obtain the dressed photon propagator is a two-step process [8]: short-range nuclear interactions are resummed into the proton contribution to the photon 

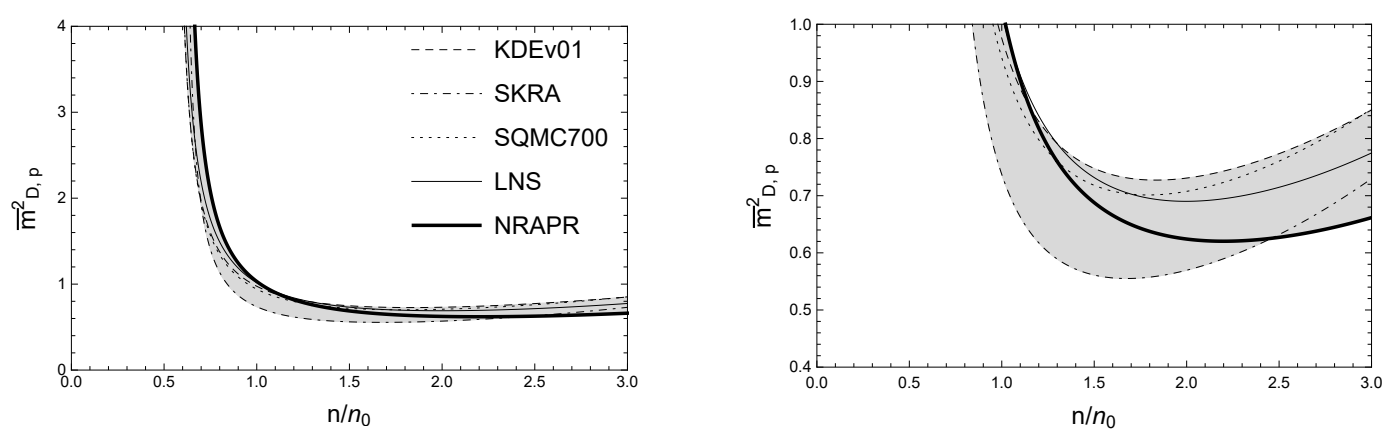

Figure 3: Density dependence of the resummed screening mass Eq. 2.6, normalized over the standard Debye mass $\bar{m}_{D, p}^{2}=\tilde{m}_{D, p}^{2} / m_{D, p}^{\prime 2}$, taken from Ref. [8]. The plots depict the rapid increase of static screening in proximity to the crust-core boundary region of neutron stars. In order to better resolve the various Skyrme models, the plot on the right-hand side enlarges a small segment around saturation density.

polarization tensor,

$$
\tilde{\Pi}_{p}=e^{2} \frac{\Pi_{p}^{\prime}\left(1+f_{n n} \Pi_{n}^{\prime}\right)}{1+f_{n n} \Pi_{n}^{\prime}+f_{p p} \Pi_{p}^{\prime}+\Pi_{p}^{\prime} \Pi_{n}^{\prime}\left(f_{p p} f_{n n}-f_{n p}^{2}\right)},
$$

where $\Pi$ is either $\Pi_{L}$ or $\Pi_{\perp}$, and $f_{a b}$ are the residual quasiparticle interactions in the densitydensity or current-current channel. The resummed polarization tensor $\tilde{\Pi}$ then replaces the "bare" proton loop in the RPA resummation, i.e., $\Pi=\Pi_{e}+\Pi_{\mu}+\tilde{\Pi}_{p}$. As far as electromagnetism is concerned, $\Pi$ and $\tilde{\Pi}$ are consequently both of order $\alpha_{f}$; the primed quantities $\Pi^{\prime}$ are identical to the one-loop polarization functions $\Pi$, with the exception that they contain no further factors of $e^{2}$. Since induced interactions are mediated by protons, they are virtually negligible for transverse scattering. In the longitudinal channel, on the other hand, one should expect a sizable impact. Before turning to a discussion of the numerical results, it is instructive to investigate the static limit of Eq. 2.5. As usual, $\tilde{\Pi}_{L}$ yields the screening mass

$$
\tilde{m}_{D, p}^{2}=-\tilde{\Pi}_{L, p}\left(q_{0}, \mathbf{q} \rightarrow 0\right)=e^{2} \frac{m_{D, p}^{\prime 2}\left(1+m_{D, n}^{\prime 2} f_{n n}\right)}{1+m_{D, n}^{\prime 2} f_{n n}+m_{D, p}^{\prime 2} f_{p p}+m_{D, p}^{\prime 2} m_{D, n}^{\prime 2}\left(f_{p p} f_{n n}-f_{n p}^{2}\right)},
$$

with the standard definition of the Debye mass $m_{D}^{\prime 2}=\mu k_{f} / \pi^{2}$. The density dependence of the screening mass is depicted in Fig. 3, and shows a divergence at lower densities. In fact one can show that the denominator of Eq. 2.6 coincides with the condition for stability of homogeneous nuclear matter [8]. It is not surprising that the screening mass is sensible to a phase transition at the crust-core boundary; after all, it can be obtained from a second order derivative of the energy density of nuclear matter with respect to proton and neutron densities $n_{p}$ and $n_{n}$. It does, however, have significant repercussions for electromagnetic scattering close to the transition.

\section{Results and Outlook}

In the following I present exemplary results, obtained from a numerical evaluation of the one-loop expression of the scattering rate Eq. 2.2. Before taking into account induced interactions we shall 

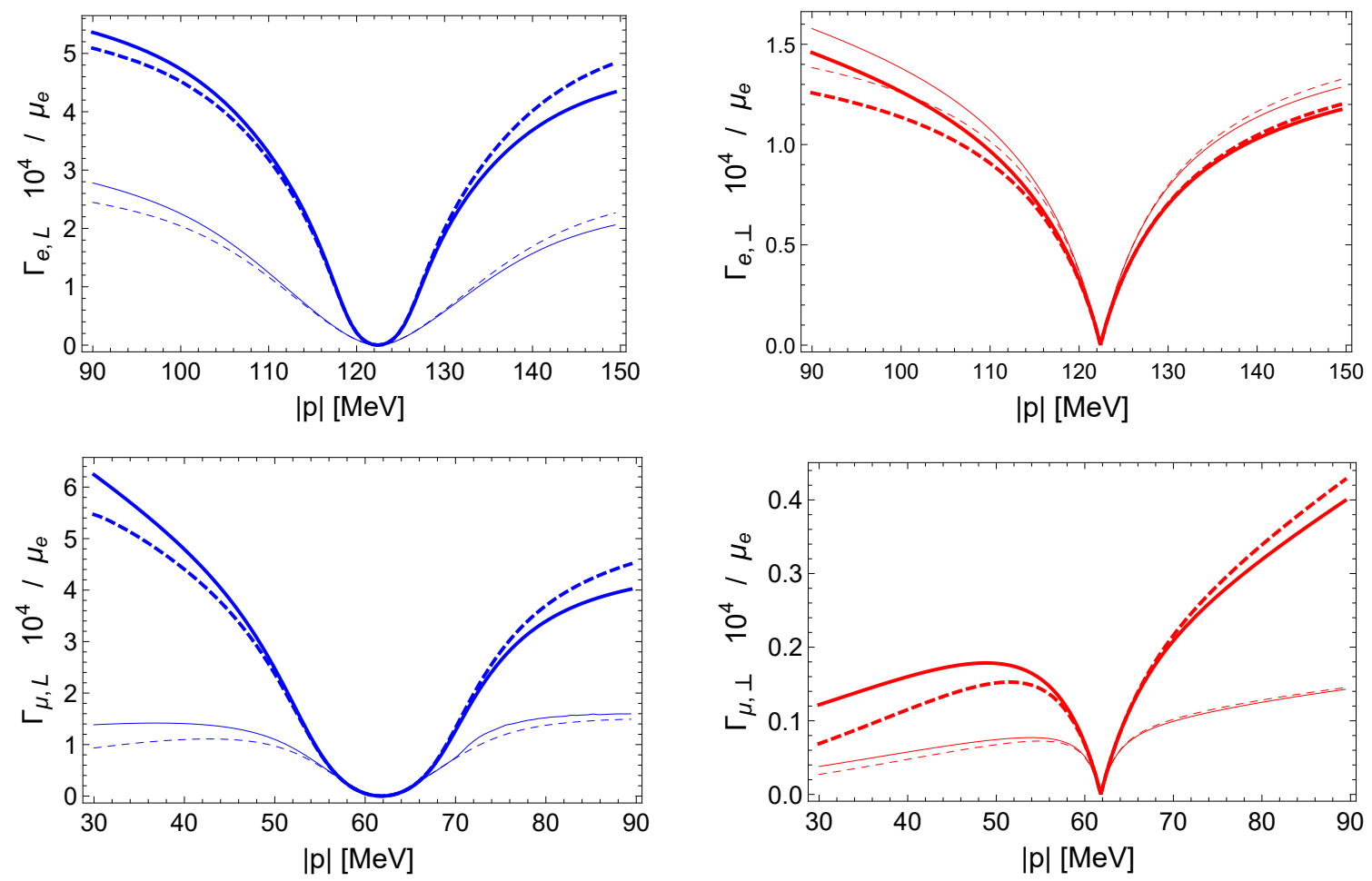

Figure 4: Total longitudinal (blue) and transverse (red) scattering rates of electrons and muons in an EMP plasma (thick), and a single-component plasma (thin). Dashed lines indicate the corresponding hard dense loop approximations. The longitudinal rate is subject to Debye screening, and the presence of heavy plasma constituents leads to a strong increase of the total rate. Electrons in the transverse channel experience the opposite effect. This happens because screening from muons and protons reduces the rate of electronelectron scattering, and this reduction outweighs the gains from additional electron-muon and electronproton scatterings. The transverse scattering rate of muons receives a sizable boost in the EMP plasma, mainly because muon-electron scattering becomes available.

take a look at pure electromagnetic scattering in an EMP plasma at saturation density. Figure 3 shows, that the ratio $\tilde{m}_{p} / m_{p}^{\prime} \sim 1$ at saturation density for all tested parameter sets except SKRA, and the impact of induced interactions should be comparatively mild. Using NRAPR Skyrme parameters the chemical potentials in $\beta$ equilibrium calculate to $\mu_{e}=\mu_{\mu} \simeq 122.4 \mathrm{MeV}, \mu_{p} \simeq 589.2$ $\mathrm{MeV}$, and the proton effective mass calculates to $m_{p}^{*} \simeq 575.3 \mathrm{MeV}$. The scattering rates of electrons and muons as function of their momenta $\mathbf{p}$ are shown in Fig. 4, and compared to scattering in a single-component plasma. In each case the rates $\Gamma_{L}$ and $\Gamma_{\perp}$ are exactly zero at $|\mathbf{p}|=k_{f}$, for $|\mathbf{p}|>k_{f}$ and $|\mathbf{p}|<k_{f}$ they correspond to the scattering of particles and holes respectively. The energy transfer $q_{0}=\varepsilon_{\mathbf{p}}-\varepsilon_{\mathbf{p}^{\prime}}$ is typically of the order of the temperature, and thus only a tiny region of $\left(k_{f} \pm 1\right) \mathrm{MeV}$ is relevant for scattering processes in neutron star matter. For comparison the hard dense loop (HDL) approximation of $\Gamma$ is shown, which extracts the leading contribution to Eq.2.4 for $q_{0} \ll \mu$ and $|\mathbf{q}| \ll k_{f}$. In close proximity to the Fermi surface the HDL result agrees remarkably well with the full one-loop result.

The impact of induced interactions on the damping rate of electrons is displayed in Fig. 5, for 

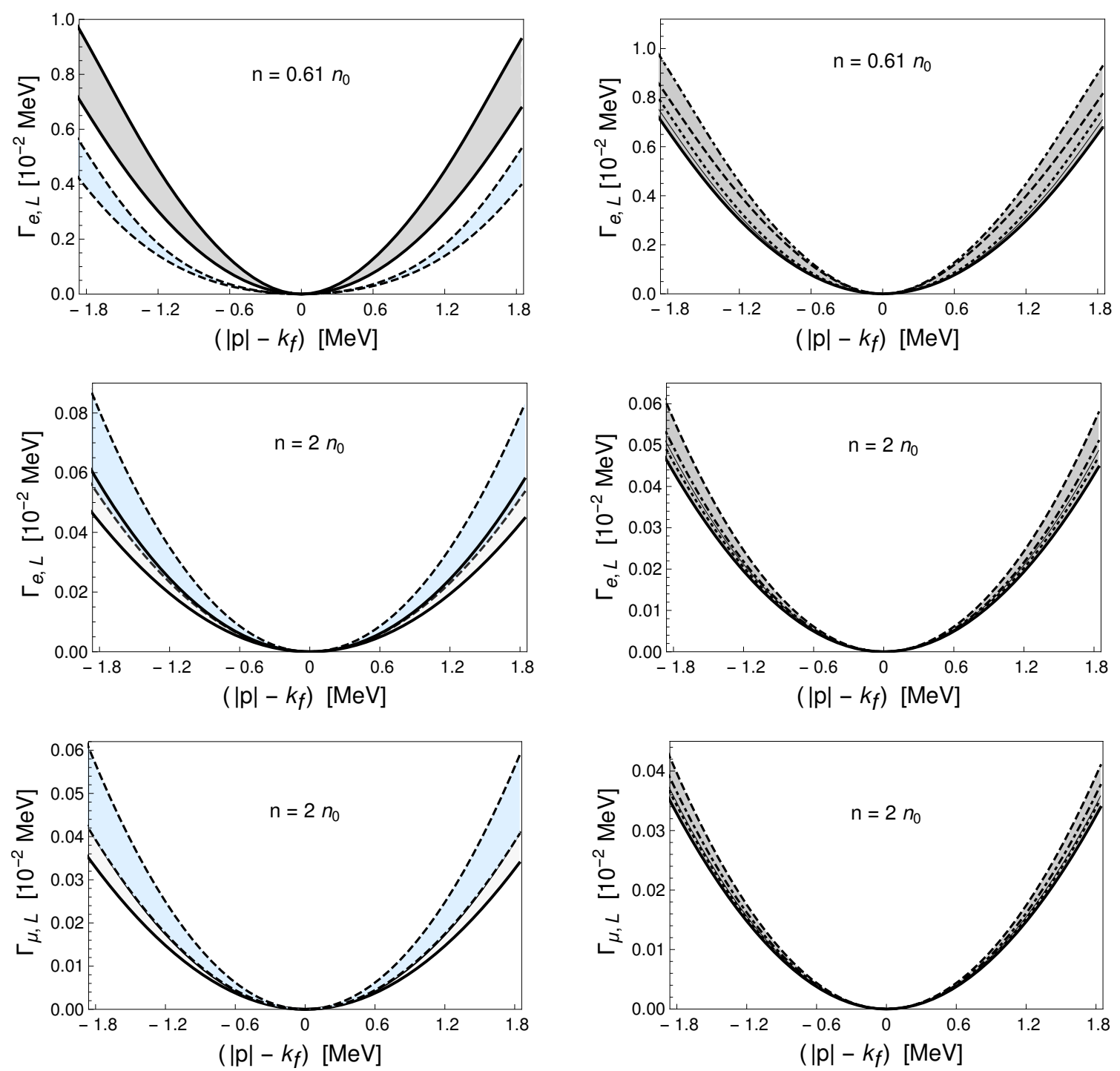

Figure 5: Longitudinal scattering rates of electrons close to the crust-core boundary, and of electrons and muons deeper inside the core. The gray and blue bands depict the results for $\Gamma_{L}$ with and without induced interactions respectively, the panels on the right-hand side resolve the various Skyrme models that constitute the gray band. Each model predicts a slightly different value for $k_{f}$ in $\beta$ equilibrium, and the rates are computed for small deviations of $\mathbf{p}$ around these values. To facilitate the comparison of the various models, the rates are not normalized over $\mu_{e}$ as in Fig. 4 . The relatively broad width of the gray band stems predominantly from the relative distance of $n$ to the critical density $n_{c}$ in each model, the Skyrme forces per se are well constrained at lower densities. The impact of induced interactions at $n=0.61 n_{0}$ is substantial. Note further, that the scattering rate at $n=0.61 n_{0}$ is almost two orders of magnitude larger than at $n=2 n_{0}$. Deeper inside the core the scattering rates of electrons and muons are almost identical in magnitude. The muon rate, however, remains small upon decreasing the density, mainly because the muon fraction quickly falls below $1 \%$. 
two densities $n \sim 0.6 n_{0}$ and $n=2 n_{0}$. The first value is chosen such that all Skyrme parameters used in Fig. 2.6 support stable homogeneous nuclear matter, and lies below the threshold at which muons appear. In this region screening effects of protons are dramatically increased. The second value corresponds to a region deeper in the core, where the screening effects of protons are slightly reduced compared to pure electromagnetic Debye screening. The bands shown in Fig. 5 encompass the results from all five parameter sets, computed for small particle/hole energies around $k_{f}$, and clearly demonstrate the impact of induced interactions. In general the results show a strong increase of the scattering rate upon approaching the crust-core boundary, with and without induced interactions.

The fact that induced interactions primarily modify longitudinal scattering, makes them particularly relevant for the damping rates of heavy particles. The absence of muons at lower densities leaves protons as the only heavy particles that may engage directly into electromagnetic scattering. To get an idea how their damping rates are modified at the edge of stability, two of the Skyrme models, NRAPR and SQMC700, with almost identical critical densities, are employed in the calculation of $\Gamma_{e}$ and $\Gamma_{p}$. The results are displayed in Fig. 6. Without induced interactions one finds the familiar picture; the scattering rate is completely dominated by electrons. Including induced interactions boosts the proton scattering rate, which then reaches up to $20 \%$ of the electron rate. Note, that the present computation is based on Eq. 2.2, and consequently assumes that protons scatter initially via the exchange of photons and plasmons.

Naturally there are other channels in which protons, and even more importantly, neutrons, initially interact via strong forces. In each of these channel, screening effects will play an important role. Placing an emphasize on the outer core of neutron stars, a systematic study of the scattering rates of leptons and nucleons, taking into account correlations of electromagnetic and strong interactions, should be carried out. The results will be particularly relevant for the ongoing efforts to find efficient damping mechanisms for r-modes. The study presented in this article is based on a very "conservative" model of matter inside the outer core of neutron stars; it does not consider phases of inhomogeneous nuclear matter, or the superconductivity of protons, often associated with this density region. It is certainly interesting to ask, whether such a simple manifestation of nuclear matter could in principle exhibit a large enough visocity to damp r-modes, and stabilize fast spinning stars. Finally, if proton superconductivity is taken into account, the transverse photon is massive due to the Meissner effect, which suppresses electron-electron, electron-muon, and electron-proton scattering. The impact of induced interactions on scattering rates in superconducting nuclear matter thus warrants further study.

\section{Acknowledgements}

The author has been supported by an Erwin Schroedinger fellowship of the Austrian science foundation FWF, project no. J3639.

\section{References}

[1] A. Schmitt and P. Shternin, Reaction rates and transport in neutron stars, arXiv:1711.06520 [astro-ph.HE]. 

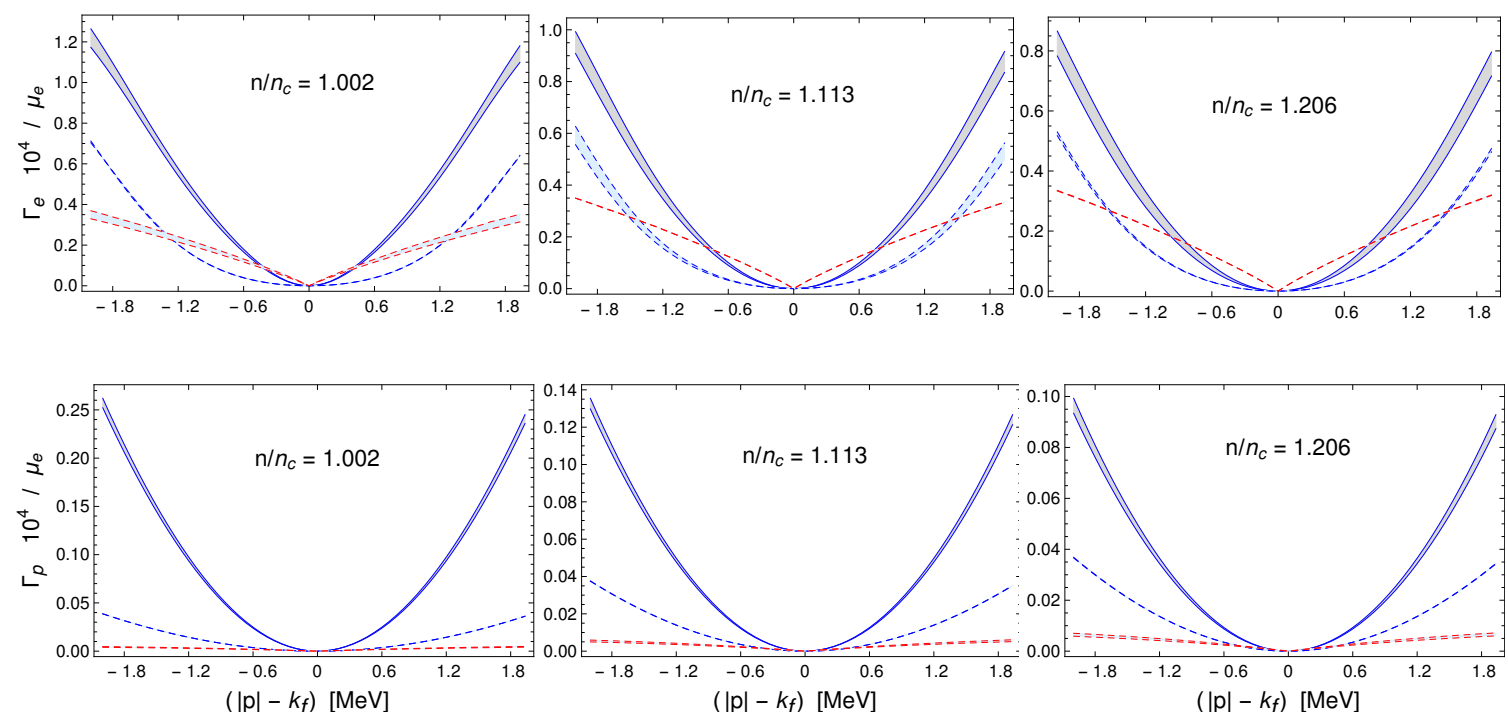

Figure 6: Electromagnetic scattering of electrons and protons close to the crust-core boundary, computed using NRAPR and SQMC700 Skyrme parameters. Both models exhibit similar critical densities, $n_{c} \sim$ $0.54 n_{0}$. The rates are normalized over the same chemical potential, $\mu_{e} \sim 87.2 \mathrm{MeV}$. Solid and dashed blue lines depict the longitudinal rates with and without induced interactions respectively (the latter coincide almost perfectly in both models), dashed red lines depict the transverse rates, for which induced interactions can safely be ignored. The three densities cover the region in which muons are absent. While the contribution of protons to electromagnetic scattering is still small compared to the contribution of electrons, the impact of induced interactions is striking. The results confirm once more, that scattering of heavy particles is dominated by the exchange of longitudinal plasmons.

[2] A. Sedrakian and J. W. Clark, Superfluidity in nuclear systems and neutron stars, arXiv:1802.00017 [nucl-th].

[3] P. S. Shternin and D. G. Yakovlev, Electron thermal conductivity owing to collisions between degenerate electrons, Phys. Rev. D 74, 043004 (2006) [astro-ph/0608371].

[4] P. S. Shternin and D. G. Yakovlev, Shear viscosity in neutron star cores, Phys. Rev. D 78, 063006 (2008) [arXiv:0808.2018 [astro-ph]].

[5] P. Shternin, Transport coefficients of leptons in superconducting neutron star cores, Phys. Rev. D 98, no. 6, 063015 (2018) [arXiv:1805.06000 [astro-ph.HE]].

[6] E. Flowers and N. Itoh, Transport properties of dense matter, APJ 206 (1976)

[7] B. Bertoni, S. Reddy and E. Rrapaj, Electron-neutron scattering and transport properties of neutron stars, Phys. Rev. C 91, no. 2, 025806 (2015) [arXiv:1409.7750 [nucl-th]].

[8] S. Stetina, E. Rrapaj and S. Reddy, Photons in dense nuclear matter: Random-phase approximation, Phys. Rev. C 97, no. 4, 045801 (2018) [arXiv:1712.05447 [astro-ph.HE]].

[9] N. Andersson, A New class of unstable modes of rotating relativistic stars, Astrophys. J. 502, 708 (1998) [gr-qc/9706075].

[10] H. Heiselberg and C. J. Pethick, Transport and relaxation in degenerate quark plasmas, Phys. Rev. D 48, 2916 (1993). 